\title{
Figuras de ancho constante: un tema por explorar
}

\author{
Óscar Javier Molina Jaime* \\ Leidi Cristina Gil Fuentes* \\ Martha Helena Orjuela Gómez****
}

Artículo recibido: 15-01-2012 y aprobado: 15-11-2012
Resumen: El objetivo de este artículo es describir algunos métodos de construcción de figuras de ancho constante en el entorno Cabri Geometry II Plus; tales métodos son producto de un estudio sobre algunas propiedades y la historia de figuras con esta característica. Pretendemos, específicamente, presentar un contexto que provee información sucinta de propiedades básicas. Exponemos métodos de construcción que las generan describiendo los caminos que se utilizaron para deducirlos; además, mostramos algunas aplicaciones que se les han dado a las figuras de ancho constante en contextos matemáticos que favorecen la construcción de otro tipos de figuras, a saber, curvas de Zindler.

Palabras clave: Figuras de ancho constante, construcción y Cabri Geometry II Plus.

\section{Constant width Figures: A subject to explore ${ }^{1}$}

Abstract: The goal of this paper is to describe some methods of construction of constant width figures on Cabri Geometry II Plus environment; such methods are the study result of some properties of figures with this property. Methods of construction that allow generate them and the way to deduce these methods are shown. Moreover, we present some of their applications on mathematics contexts that permit the construction of other figures, namely, Zindler curves.

Keywords: Figures of constant width, construction and Cabri Geometry II Plus.

\footnotetext{
* Universidad Pedagógica Nacional: ojmolina@pedagogica.edu.co

** Universidad Pedagógica Nacional: leidifuentes@yahoo.es

*** Universidad Pedagógica Nacional: tuchisgomez@hotmail.com
}

\footnotetext{
${ }^{1}$ Una versión preliminar de este artículo ha sido publicada en las memorias del XX Encuentro de Geometría y sus aplicaciones, Universidad Pedagógica Nacional, Bogotá, 23 a 25 de junio de 2011.
} 


\section{Introducción}

En el transcurso de nuestra experiencia académica, tanto en la secundaria como en la universidad, el estudio de la geometría se centró en algunas propiedades de figuras geométricas planas como triángulos, cuadriláteros y circunferencias, así como de algunos cuerpos sólidos (esferas, cilindros, poliedros). Esta experiencia nos condujo, y creemos que no somos las únicas personas con esa característica, a la idea equivocada de que solo las circunferencias (y las esferas) son figuras con la propiedad de ancho constante. Sin embargo, existen otras que tienen esta propiedad pero que no forman parte de las temáticas usuales a estudiar en la formación escolar o universitaria.

Pero, ¿qué es una figura de ancho constante? Las figuras geométricas de ancho constante son aquellas que tienen el mismo ancho en cualquier dirección; el ancho de una figura se refiere a la distancia entre dos rectas perpendiculares a la dirección dada que aprisionan dicha figura (Montejano, 1998). Las rectas que aprisionan a la figura se deben entender como rectas que la intersecan en solo un punto (es el caso de las rectas tangentes a la figura) y que son perpendiculares a una cuerda que indica la dirección. Si los extremos de esta cuerda están contenidos, uno en cada una de las rectas, la magnitud de esta cuerda indica el ancho de la figura.

El anterior panorama nos llevó a indagar sobre la literatura existente en relación con tal temática; sin embargo, fue poca la documentación encontrada en el marco de las matemáticas básicas, sí en el contexto de la topología en un nivel superior (conjuntos convexos). Quisimos, entonces, desarrollar un estudio que además de precisar otras figuras, diferentes a la circunferencia, que cumplan con esa propiedad, presente información sobre la evolución histórica del estudio de estas figuras, explicite algunas de sus propiedades geométricas (por ejemplo, en relación con sus diámetros, normales, convexidad, perímetros, áreas), sus aplicaciones en contextos matemáticos o de la realidad y procedimientos de construcción en un entorno de geometría dinámica. Precisamente, esta última intención se corresponde con la pregunta sugerida por Alsina (2005), en relación con cómo generar infinitas clases de figuras de ancho constante; este autor particulariza dicha intención al caso de cómo generarlas a partir del triángulo de Reuleaux (más adelante se precisará la definición de esta figura).

En este sentido, presentamos un documento que desarrolla parte de nuestras intenciones; específicamente, usamos un entorno de geometría dinámica como medio que facilita la producción de procedimientos de construcción de estas figuras. Un entorno como este no solo permite ilustrar propiedades fundamentales de figuras de ancho constante mediante representaciones gráficas, sino que también se convierte en una herramienta que favorece la identificación o estudio de tales propiedades; lo anterior en tanto que un entorno como este facilita la visualización y permite la exploración experimental, y la modificación continua de las construcciones, con lo que se obtienen, fácil y casi inmediatamente, numerosos ejemplos con una sola figura. 
Al realizar un estudio como el pretendido, creemos que contribuimos con documentación que a posteriori será de utilidad en la realización de propuestas didácticas enfocadas en estas temáticas no usuales pero interesantes.

\section{Información general de las figuras de ancho constante}

Las figuras geométricas de ancho constante son aquellas que tienen el mismo ancho en cualquier dirección; el ancho de una figura se refiere a la distancia entre dos rectas paralelas entre sí y perpendiculares a la dirección dada; estas rectas se conocen como rectas soporte, las cuales intersecan a la figura en un solo punto y son perpendiculares a una cuerda de la figura que indica dicha dirección (Montejano, 1998). Si los extremos de esta cuerda están contenidos, uno en cada una de las rectas soporte, la magnitud de esta cuerda indica el ancho de la figura.

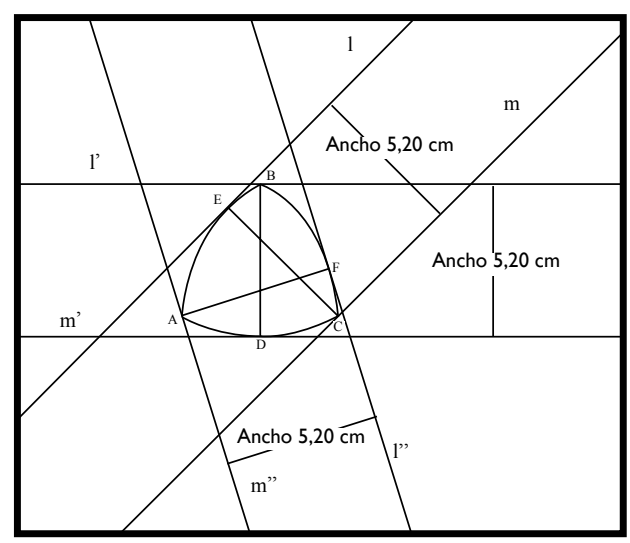

Figura1. Diagrama de ancho constante.Triángulo de Relaux

Las figuras de ancho constante han estado presentes a lo largo de la historia en diversos contextos como el arte, la mecánica y la ingeniería. Para ejempli- ficar esto podemos hacer referencia a las monedas británicas, las cuales están diseñadas con figuras de ancho constante construidas a partir de un polígono regular de lado impar, ya que gracias a su forma evitan que las máquinas expendedoras se bloqueen, pues éstas miden la anchura de la moneda que es introducida en ellas (Corbalán, 2007). Otra de las aplicaciones se puede apreciar en las tapas de las alcantarillas en forma de triángulo de Reuleaux que se encuentran en la ciudad de San Francisco, en California, el diseño utilizado en estas tapas evita que estas caigan a través del propio agujero sin importar como se orienten y además se ahorra en material de fabricación puesto que el triángulo de Reuleaux tiene menor área que un círculo del mismo diámetro. Por otro lado, las figuras de ancho constante han sido usadas también en la arquitectura y en el diseño de varios objetos como lápices triangulares, púas de guitarra, relojes y llantas de una bicicleta (esto último inventado por el chino Guan Baihua, ver figura 2.

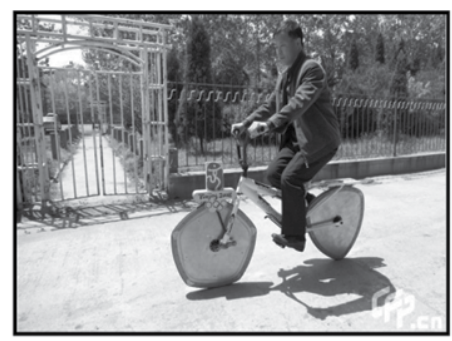

Figura 2. Bicicleta de Guan Baihua

Para finalizar este apartado, vale la pena precisar que el triángulo de Reuleaux es quizás la figura de ancho constante más utilizada para las aplicaciones de la ingeniería o, por lo menos, es a la que más se hace referencia en la bibliografía consultada. Ha sido pieza 
clave en algunos diseños, accesorios de máquinas, como el motor Wankel (motor de combustión interna), la broca que perfora agujeros casi cuadrados, inventada por Harry James Watts en 1914 (Montejano, 1998). Aunque esta broca esté basada en un triángulo de Reuleaux porque puede girar dentro de un cuadrado, vale mencionar que cualquier figura de ancho constante puede circunscribirse a un cuadrado que tenga la longitud de sus lados igual a la longitud del ancho de la figura en cuestión y, por tanto, puede girar dentro de él.

\section{Métodos de construcción de figuras de ancho constante}

Existen varios métodos de construcción de las figuras de ancho constante; en esta sección presentamos algunos de ellos. Los métodos presentados se basan inicialmente en la construcción del triángulo de Reuleaux a partir de su definición; seguidamente mostramos la construcción de dichas figuras partiendo de polígonos regulares e irregulares, haciendo uso de hipocicloides y evolutas ${ }^{1}$ en la construcción de la curva de ancho constante de Euler. También realizamos construcciones a partir de figuras de ancho constante construidas con anterioridad con alguno de los métodos expuestos. Por último, exponemos la construcción de una figura de ancho constante a partir de una curva con características especiales, las cuales se evidencian más adelante.

\section{Sobre el triángulo de Reuleaux}

La figura de ancho constante más utilizada es el triángulo de Reuleaux al

1 Es el lugar geométrico de todas las normales a una figura. cual nos referiremos más adelante; sin embargo fue Euler en 1778 el primero en estudiar las propiedades de estas figuras; él las conocía como orbiformes, que significa, "curvas con forma de círculo" (Montejano, 1998).

Para iniciar este apartado, mostramos el método de construcción del triángulo de Reuleaux (figura de ancho constante presentada en 1976 por el ingeniero y matemático Franz Reuleaux) generado a partir de su definición. Específicamente,

Dado un triángulo equilátero de lado L, untriángulo de Reuleauxes la unión de los tres arcos de circunferencia cuyos extremos son dos vértices del triángulo, el centro de la circunferencia es el vértice restante y el radio de la misma es $L$.

El procedimiento se ilustra en la figura 3.

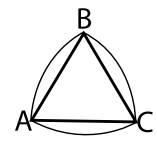

a. Construimos un triángulo equilátero $A B C$.

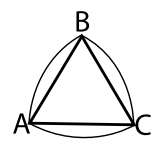

c. Construimos $\hat{A B}, \hat{B C}$ y $\hat{A C}$.

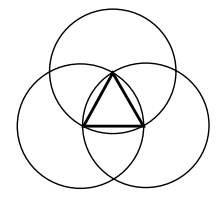

b. Construimos

$\odot A_{-}(, A B$,

$\odot B_{-}(, A B) y$

$\odot C_{-}$(,CA).

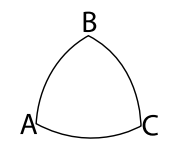

d. Ocultamos las circunferencias y el triángulo. $\hat{\Delta} \mathrm{ABC}$.
Figura 3. Triángulo de Reuleaux $\hat{\triangle} \mathrm{ABC}$

Realizando algunas construcciones auxiliares a la figura anterior en Cabri, es posible ilustrar que el triángulo de 
Reuleaux, el cual denotamos $\hat{\Delta} \mathrm{ABC}$, es una figura de ancho constante; las construcciones auxiliares se basan en la construcción de las rectas soporte, paralelas entre sí y perpendiculares a una dirección dada y en mostrar que el ancho de la figura en cualquier dirección es el mismo (figura 1). A continuación presentamos la siguiente justificación basada en Montejano (1998) de que el triángulo de Reuleaux es una figura de ancho constante. La manera en que justificamos esta construcción es la utilizada para demostrar los demás procedimientos presentes en este documento, por ello omitimos dichas justificaciones.

\section{Demostración de que el $\widehat{\triangle} A B C$ es una figura de ancho constante}

Sea la dirección de una cuerda de $\hat{\Delta} \mathrm{ABC}$ que contiene a uno de los vértices de $\hat{\Delta}$ $\mathrm{ABC}$. Digamos que $\mathrm{CD} "=\mathrm{d}$ donde $\mathrm{D} " \epsilon$ $\widehat{\mathrm{AB}}$, entonces existe la recta $\mathrm{n}, \mathrm{n} \perp \overline{\mathrm{CD}^{\prime \prime}}$ por $\mathrm{D}$, esto por el teorema de la recta tangente a una circunferencia, y sea la recta $\mathrm{n}$ tangente a $\widehat{\mathrm{AB}}$ por el punto $\mathrm{D}^{\prime \prime}$; tenemos que la recta perpendicular a la recta $\mathrm{n}$ por el punto $\mathrm{D}$ " contiene el punto C que es centro de $\widehat{A B}$ (figura 4).

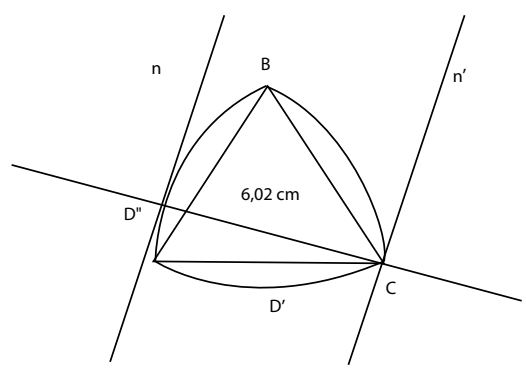

Figura 4. Construcción de rectas soporte a un $\Delta \hat{\Delta} \mathrm{ABC}$

Por otro lado, la recta $n^{\prime} \perp \overline{\mathrm{CD}^{\prime \prime}}$ por $\mathrm{C}$ es paralela a $n$ y además n' es una recta soporte a $\hat{\Delta} \mathrm{ABC}$ ya que esta recta interseca a $\hat{\Delta} \mathrm{ABC}$ sólo solo en el punto $\mathrm{C}$ y la deja contenida en un solo semiplano. El ancho de $\widehat{\triangle} \mathrm{ABC}$ en la dirección d está dado por la distancia entre $n$ ' y $n$, que es $C D$ " ya que las rectas n' y $n$ son paralelas entre sí y perpendiculares a la cuerda $\overline{\mathrm{CD}}$ ". Luego, por la definición de $\hat{\Delta} \mathrm{ABC}$ y dado que $\overline{C^{\prime \prime}}$ es un radio de $\odot \mathrm{C}(\mathrm{AB})$, tenemos que en cualquier dirección los radios de las circunferencias que tienen como centro los puntos A y B tienen la misma longitud y son el ancho de $\hat{\Delta}$ ABC. Podemos concluir que el ancho en cualquier otra dirección es $\mathrm{CD}^{\prime \prime}$ siendo $\mathrm{CD} "=\mathrm{AB}$.

\section{Figuras de ancho constante, partiendo de polígonos regulares}

A partir del método de construcción del triángulo de Reuleaux y de su definición, nos surgieron los siguientes interrogantes: ¿Se originan figuras de ancho constante si en lugar de utilizar un triángulo equilátero, utilizamos cualquier polígono regular? Si no es así, ¿Qué cambios podríamos plantear en el método para obtener figuras con esta propiedad? Gracias a la característica esencial de Cabri, su dinamismo producto de la función de arrastre, logramos dar respuesta a esos interrogantes y encontrar generalidades que nos permitieron obtener figuras de ancho constante. Por ejemplo, al partir de polígonos regulares de un número impar de lados, observamos que utilizando el mismo método de construcción para el triángulo de Reuleaux, obtenemos figuras de ancho constante de manera que su ancho es igual a la longitud del diámetro del polígono. En la figura 5 se puede observar el procedimiento para generar la figura de ancho constante $\varphi \mathrm{ABCDE}$ obtenida a partir de un pentágono. 


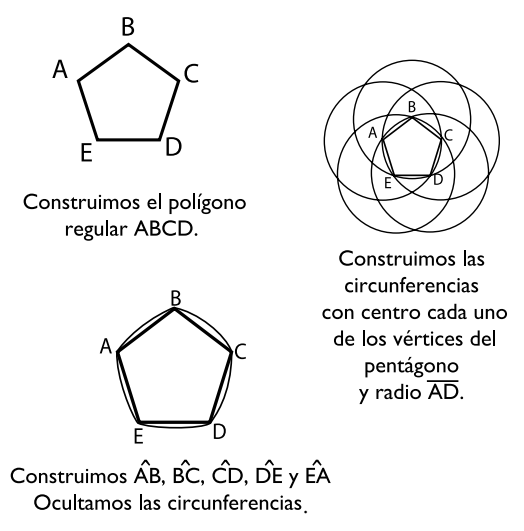

Figura 5. Pentágono de ancho constante

Por otro lado, con polígonos regulares de un número par de lados se mostró que el método de construcción anterior no genera figuras de ancho constante, ya que en cualquier dirección el ancho no es el mismo (figura 6).

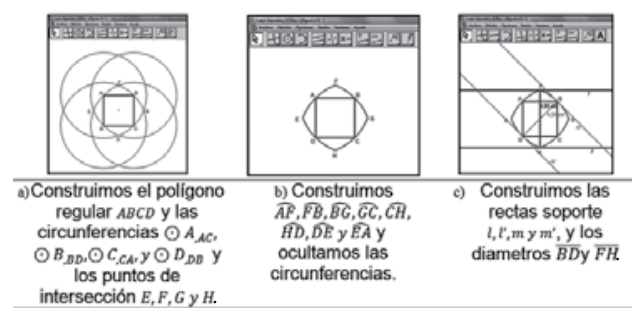

Figura 6. Cuadrado de ancho no constante

Pero al realizar unas construcciones auxiliares en el método anterior, se pueden obtener figuras de ancho constante, no solo una por polígono, sino varias dependiendo del número de lados del mismo; para estas figuras el ancho sigue siendo igual a la longitud del diámetro del polígono, básicamente las construcciones auxiliares consisten en construir nuevas circunferencias con radio igual a la longitud del diámetro del polígono y centro en los puntos de intersección de las circunferencias que tienen el mismo radio, pero el centro en los vértices del polígono. La figura de ancho constante se obtiene a partir del cuadrado (figura 7).

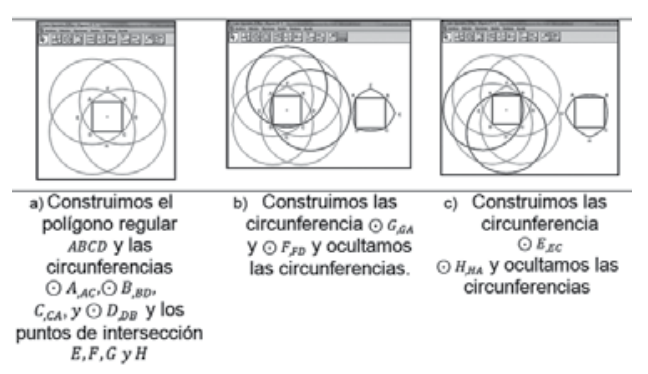

Figura 7. Cuadrados de ancho constante

\section{Curva de ancho constante de Euler}

$\mathrm{Al}$ indagar en la historia de las figuras de ancho constante, se logró establecer otra figura de ancho constante llamada "curva de ancho constante de Euler". Montejano (1998) afirma que esta curva surge de la hipocicloide de Steiner (aquella que se obtiene como la órbita de un punto de un círculo de radio $\mathrm{r}$ mientras rueda dentro de un círculo de radio 3r) y su evoluta. A partir de esta idea, y luego de realizar una exploración en Cabri, logramos construir la figura de ancho constante. Para poder construir la curva de ancho constante, fue necesario construir primero la hipocicloide de Steiner, que se realizó partiendo de una circunferencia de radio $r$ y un punto sobre ella, de la transferencia de la medida de arcos y la medida de la tercera parte del radio de la circunferencia y encontrando un lugar geométrico (figura 8). 


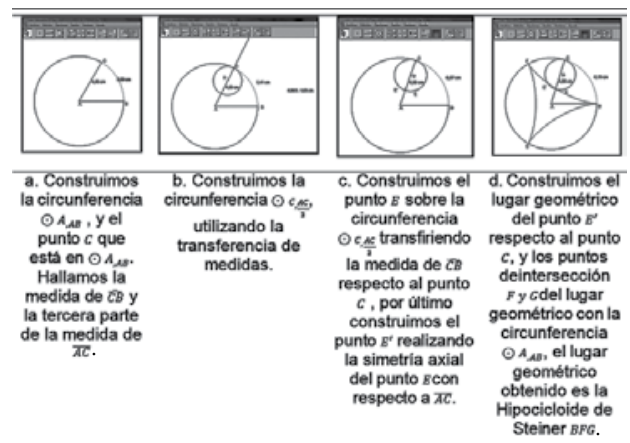

Figura 8. Hipocicloide de Steiner BFG

En seguida, para construir la curva de Euler, se construyó la evoluta de dicha hipocicloide BFG, a partir del lugar geométrico de todas las rectas normales a la hipocicloide (figura 9).

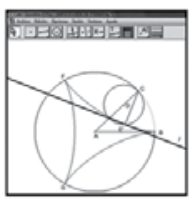

a. Construimos la hipocicloide de Steiner, el segmento $\overline{E^{\prime} C}$ y la recta $l \perp \overline{E^{\prime} C}$ que pasa por $E^{\prime}$.

Figura 9. Evoluta de la hipocicloide de Steiner HIJ

Teniendo la hipocicloide de Steiner y realizando algunas construcciones auxiliares, rectas, circunferencias, puntos de intersección, lugares geométricos que se explicitan en la figura 10, se pudo construir la curva de ancho constante de Euler.
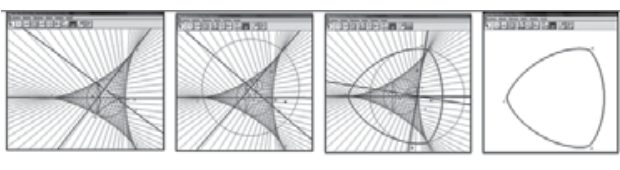

a. Construimos la

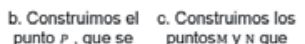

Evoluta de la punto $P$, que se puntosm y $N$ que Hipocicloide de

teiner. $F G$, su punto medio que

enotaremos con $K y$

la recta $n$ que contiene a los
puntos / y $K$. $\begin{array}{cc}\text { origina de } & \text { son los puntos de } \\ \text { laintersección } & \text { intersección de }\end{array}$ entre la recta $n y \quad \mathrm{OE}_{n}$ con la recta m el lugar $B F G . \quad y$ construimos los Construimos lugares de los puntos M Y $\mathrm{N}$ que dependen del
punto $\mathrm{E}$.

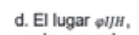

d. El lugar wJjh,
es la curva de Euler

Figura 10. $\varphi \mathrm{HIJ}$, curva de ancho constante de Euler
Como podemos observar, la curva de ancho constante de Euler se parece al triángulo de Reuleaux, pero gracias a las características que tiene el software Cabri, como la visualización y la exploración, se pudo establecer que la curva de ancho constante no cumple con la definición del triángulo de Reuleaux; esta exploración, con el propósito de verificar, consistió básicamente en construir el triángulo equilátero JHI y, a partir de este, elaborar el triángulo de Reuleaux, de esta manera observamos que los arcos que componen el triángulo de Reuleaux $\hat{\Delta}$ JHI no se corresponden con la curva de Euler HIJ.

\section{Figura de ancho constante: ¿otra forma de construir la curva de Euler?}

A continuación mostramos una figura de ancho constante, generada a partir de un triángulo equilátero y del segmento que cumple con la propiedad de ser tangente a los arcos simétricos de los arcos que conforman un triángulo de Reuleaux, con respecto a cada uno de los lados del triángulo equilátero (figura 11). Es importante resaltar que la figura generada a partir de este método tiene un parecido a la curva de ancho constante de Euler, pero no contamos con las herramientas teóricas para establecerlo. Empíricamente se conjeturó que ello es válido, superponiendo las respectivas representaciones.

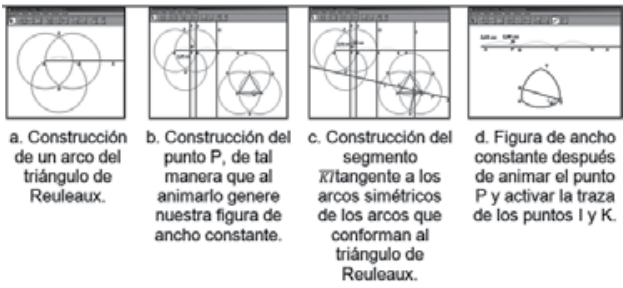

Figura 11. Figura de ancho constante a partir de un triángulo equilátero $\varphi \mathrm{IJ} H$ 
Para este caso, el mismo método de construcción permite inferir que la figura $\varphi R S T$ tiene ancho constante, ya que la medida del ancho de la figura depende de la longitud de un segmento, que para este caso es $\overline{\mathrm{KI}}$, el cual tiene la misma longitud en cualquier dirección.

\section{Figuras de ancho constante a partir de las figuras de ancho constante obtenidas}

Otro de los métodos utilizados para construir figuras de ancho constante consiste en partir de otras figuras con esta propiedad. El método se basa en Rademacher y Töeplitz (1990) y consiste en redondear las puntas de las figuras de ancho constante obtenidas anteriormente, mediante el uso de arcos de circunferencia; de esta manera obtenemos figuras de ancho constante sin puntas. El procedimiento se ilustra en la figura 12 partiendo del triángulo de Reuleaux.
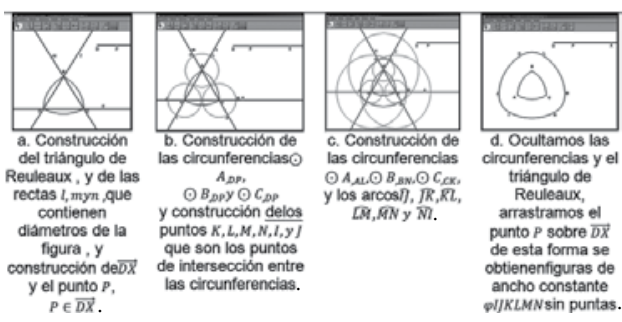

Figura12. Figuras de ancho constante a partir de otra figura de ancho constante

Para construir figuras de ancho constante, utilizando el método anterior y partiendo de cualquier otra figura con dicha propiedad, es necesario construir inicialmente las rectas que contienen dos vértices de la figura de ancho constante que se toma como base para la construcción, los cuales son extremos de un diámetro de dicha figura; de esta manera construimos dos rectas que contienen el mismo vértice, algunos ejemplos se pueden evidenciar en la figura 13.

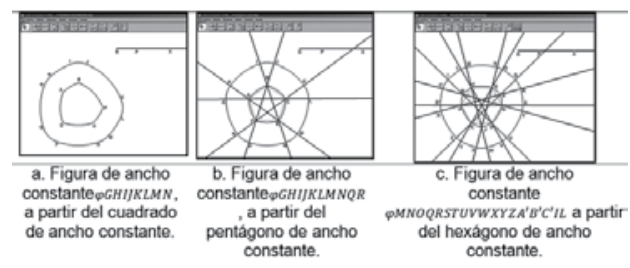

Figura13. Figuras de ancho constante a partir de otra figura de ancho constante

Hasta el momento, los métodos de construcción para generar figuras de ancho constante presentados parten de figuras que son regulares, donde los métodos de construcción son muy parecidos entre sí, se basan en la copia de los métodos de las construcciones anteriores y utilizan algunas construcciones anexas, como la construcción de arcos para redondear figuras. Nos surgió la inquietud de saber si los métodos anteriores nos podrían ser de ayuda para construir figuras de ancho constante, pero esta vez partiendo polígonos irregulares. En consecuencia, mostramos, a continuación, la figura de ancho constante obtenida es de un triángulo escaleno y, además, presentamos el método de construcción de una figura de ancho constante desde una curva convexa con unas características especiales.

\section{Construcción de una figura de ancho constante a partir del triángulo escaleno}

El método mostrado a continuación para la construcción de la figura de ancho constante a partir del triángulo escaleno (figura 14), fue propuesto por Middletown (2000, citado por Michon, 2010).

El diámetro de la figura obtenida utilizando el método descrito en el gráfico anterior es $(\mathrm{AB}+\mathrm{AC})-\mathrm{BC}$. 


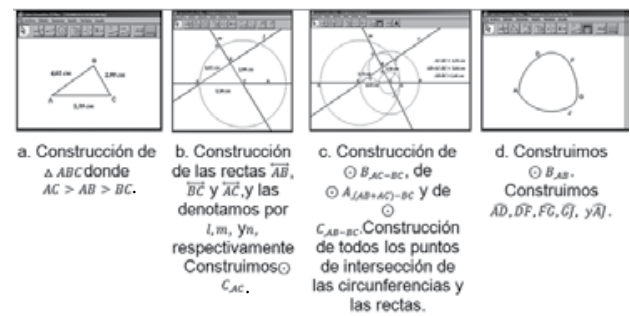

Figura14. Figura de ancho constante a partir de un triángulo escaleno

\section{Figura de ancho constante a partir de una figura convexa}

Finalmente el último método expuesto fue encontrado a partir de la construcción de una figura convexa, está construcción estuvo basada en el trabajo de Rademacher y Töeplitz (1990), quienes parten de la construcción de una curva ACB con las siguientes características: está contenida entre las rectas 1 y m salvo en los puntos A y B, la curva ACB junto con $(\mathrm{AB})$ forman una región convexa $\mathrm{y}$ por cada punto $\mathrm{T} \in \mathrm{ACB}$ existe un círculo de radio $A B$ tangente a la recta soporte de la curva $\mathrm{ACB}$ por $\mathrm{T}$ que contenga la curva ACB y se construye el lugar geométrico, generado por las circunferencias que tienen como centro los vértices de la curva con características especiales, y como radio la medida del segmento que contiene dicha curva (figura 15).

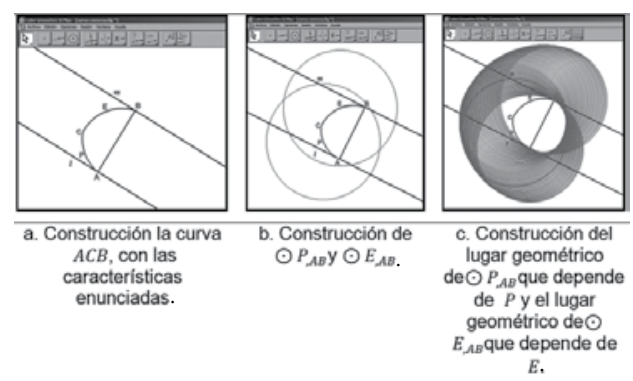

Figura15. Figuras de ancho constante a partir de una figura convexa
La figura formada por la curva ACB y la frontera que delimitan los lugares geométricos que se encuentra entre las rectas $\mathrm{l}$ y $\mathrm{m}$ y en el semiplano definido por $(\stackrel{\leftrightarrow}{\mathrm{AB}})$ que no contiene la curva $\mathrm{ACB}$ es una figura de ancho constante cuyo ancho es $\mathrm{AB}$.

\section{Una aplicación de las figuras de ancho constante en las matemáticas: otra construcción}

Existe una clase particular de figuras que tienen aplicación en las matemáticas, particularmente, en la división del área y el perímetro de las figuras a la mitad, estas figuras son llamadas "curvas de Zindler" y se pueden construir a partir de las figuras de ancho constante. Tomemos, por ejemplo, la figura de ancho constante $\varphi \mathrm{y}$, como en cada dirección existe un diámetro, construimos un segmento perpendicular y congruente a cada diámetro de tal manera que estos se bisequen. A medida que los diámetros de $\varphi$ vayan tomando todas las direcciones, los extremos del segmento perpendicular al diámetro describirán la frontera de una curva de Zindler (Montejano, 1998) (figura 16).

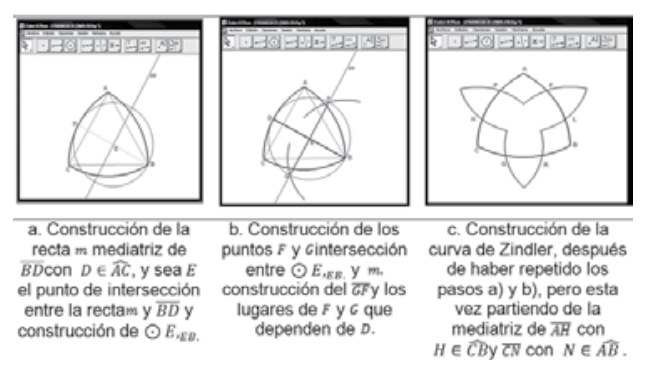

Figura16. Curvas de Zindler a partir del triángulo de Reuleaux 
El proceso puede ser revertido, es decir, se pueden construir figuras de ancho constante a partir de las curvas de Zindler, donde los segmentos perpendiculares a las cuerdas de las curvas de Zindler que dividen el perímetro y el área a la mitad serán los diámetros de la figura de ancho constante que se construirá.

\section{Conclusiones}

El principal objetivo de nuestro estudio consistió en estudiar y dar a conocer las propiedades de las figuras de ancho constante a través de la presentación de métodos para su construcción, en un entorno de geometría dinámica; además de estudiar propiedades y generalizar algunos procedimientos a partir de la exploración que nos permitía realizar el software Cabri. En este documento mostramos diversos métodos de construcción de las figuras de ancho constante haciendo uso del software de geometría Cabri II Plus, los cuales se obtuvieron a partir de: consulta de algunas de las propiedades que cumplen dichas figuras, referentes históricos de los cuales pudimos extraer la idea para construir la curva de ancho constante de Euler y de las exploraciones realizadas con este software. Cada uno de los procedimientos presentados están justificados.

Las construcciones realizadas en Cabri nos proporcionaron ideas para justificar por qué la figura construida tiene la propiedad de ancho constante debido a la relación existente entre el software y la teoría de la geometría plana euclidiana (Mariotti, 1997); en otros casos no fue necesario hacer una demostración formal puesto que el mismo procedimiento casi que validaba teóricamente la construcción. Las exploraciones consistieron básicamente en la copia de algunos procedimientos de construcción que eran válidos y la modificación de los mismos a través de ensayos para obtener figuras de ancho constante; estas exploraciones nos permitieron plantear conjeturas, específicamente la relacionada con la validez de construir figuras de ancho constante partiendo de polígonos regulares con un número impar de lados, teniendo como base el procedimiento expuesto para la construcción del triángulo de Reuleaux y de su definición. De esta manera, Cabri se convirtió en un "motor del pensamiento deductivo, pues las propiedades explícitamente construidas se convierten en premisas, siendo las conclusiones otras propiedades verificadas en la construcción” (Ministerio de Educación Nacional, 2002).

En este documento también dimos cuenta de que las figuras de ancho constante se usan en los diseños de diversos objetos; esto se hizo evidente en cada uno de los ejemplos que presentamos en la última sección, en los cuales ilustramos algunas de las aplicaciones que han tenido las figuras de ancho constante en la ingeniería, en la mecánica, la arquitectura y en las formas de algunos objetos, producto de sus propiedades que son útiles para ciertos usos.

En cuanto al software Cabri II Plus, con el cual realizamos la totalidad de las construcciones y al que no le hicimos mayor énfasis a lo largo del documento, fue pieza clave en el desarrollo de este trabajo debido a que sus características esenciales (arrastre, dinamismo y relación de la geometría dinámica con la teoría de la geometría plana euclidiana) 
favorecieron algunas actividades matemáticas como la visualización, exploración, la conjeturación y la justificación.

Para finalizar, queremos mencionar que en la realización del documento se excluyó diversa información relacionada con estas figuras puesto que la misma se alejaba de los objetivos propuestos. Por tanto se excluyó lo referente a los cuerpos de ancho constante - ya que presentan similitudes con las figuras de ancho constante -, ni se profundizó en las curvas de Zindler (la única aplicación matemática que presentamos); por esta razón, quedan aspectos que se pueden considerar en estudios posteriores como: ¿los cuerpos de ancho constante cumplen las mismas propiedades que las figuras de ancho constante? ¿Se pueden obtener dichos sólidos si se revolucionan las figuras de ancho constante? ¿Se pueden construir figuras de ancho constante con base en poliedros regulares? ¿Qué otra aplicación matemática presentan las figuras de ancho constante? ¿Existe alguna otra relación entre las curvas de Zindler y las figuras de ancho constante?

\section{Referencias}

Alsina, C. (2005). Homenaje a Reuleaux. Suma: Revista sobre Enseñanza y Aprendizaje de las Matemáticas, 48, 77- 79.

Corbalán, F. (2007). Matemáticas de la vida misma. Serie Didáctica de las Matemáticas. Barcelona: Editorial GRAÓ.

Mariotti, M. (1997). Justifying and proving in geometry: the mediation of a microworld. Proceedings of the European Conference on Mathematical Education. Praga: Prometheus Publishing House.

Michon, G. P. (2010). Geometry and Topology. Recuperado el 11 de octubre de 2009 de: http://www.numericana. com/answer/geometry.htm\#oblate

Montejano, L. (1998). Cuerpos de ancho constante. México D.F.: Fondo de Cultura Económica.

Rademacher, H. y Töeplitz, O. (1990).The enjoyment of mathematics. Selections from Mathematics for the Amateur. NuevaYork: Dover Publications Inc.

Sautoy, M. (27 de mayo de 2009). A new bicycle reinvents the wheel, with a pentagon and triangle. Timesonline. Recuperado el 2 de marzo de 2010 de: http://technology.times on line. co.uk/tol/news/tech_and_web/article6366308.ece 